\title{
Shift Register Device
}

National Cancer Institute

\section{Source}

National Cancer Institute. Shift Register Device. NCI Thesaurus. Code C50172.

An electronic circuit designed to move all data to the left or right, thereby doing multiplication, division, or parallel conversion. 